\title{
RSSI based Optimum Transmission Power Ad-Hoc on-Demand Distance Vector (rOTP-AODV) Routing Protocol
}

\author{
Amit Kumar Dogra \\ Dept. of Computer Science \& Engg. \\ Shri Mata Vaishno Devi university \\ Jammu (J\&K), India
}

\author{
Ajay Kaul, Ph.D \\ Dept. of Computer Science \& Engg. \\ Shri Mata Vaishno Devi university \\ Jammu (J\&K), India
}

\begin{abstract}
One of the major challenges faced by the Ad hoc networks is battery life of the nodes. By reducing or optimizing the energy consumed during the communication, the life of the respective nodes can be increased thereby improving the network lifetime. However the energy consumed in communication is greatly affected by the quality of link between the nodes. In this paper, we propose RSSI based Optimum Transmission Power AODV, an energy efficient routing algorithm that minimizes the energy consumed by nodes in transmission by calculating the optimal transmission power required for communication with neighboring nodes. Simulations show that incorporation of rOTP-AODV saved more than $50 \%$ of energy, along with slightly improved throughput and jitter compared to AODV.
\end{abstract}

\section{General Terms}

Ad hoc networks, AODV, rOTP-AODV, Optimum Transmission Power, TPC, Cross Layer, RSSI, Energy Efficient, Routing Protocol.

\section{Keywords}

AODV, rOTP-AODV, Optimum Transmission Power, TPC, Cross Layer, RSSI, Energy Efficient, Routing Protocol.

\section{INTRODUCTION}

Wireless Ad hoc networks are networks in which communication links are based on wireless technologies and the network structure or topology is not pre-defined rather build through dynamic network connectivity. As these nodes communicate with each other over wireless links, they have to contend with the challenges associated with radio communication, such as noise, fading, and interference. Moreover the links in wireless Ad hoc networks typically have less bandwidth than in a wired network.

With the advances in time Wireless Ad hoc networks have found application is almost every aspect of life ranging from communication, military, business, disaster management, healthcare, home automation etc and depending upon type of application, different categories of wireless Ad hoc networks have evolved. The three common subtypes of wireless Ad hoc networks are Mobile Ad hoc networks (MANETs), Wireless Sensor networks (WSNs) and Wireless Mesh Networks (WMNs).

One critical issue for the wireless Ad hoc networks is that the nodes are supported by battery which is often irreplaceable. Battery power being limited, extending the lifetime of batteries is an important issue. If a node stops operating due to loss of battery, it can result in network partitioning and there can be an interrupt in ongoing communication. Thus, the design of routing protocols for Ad hoc Networks is a challenging issue. To reduce the energy consumption in devices, there have been efforts in physical and data link layers as well as in the network layer related to the routing protocol. In last decade, substantial amount of research has been dedicated toward controlling the transmission power of a node so as to conserve energy. However most of them have focused on MAC and Physical layer to provide transmission power control (TPC). But in these approaches the Network layer and routing protocols are unable to reap the benefits associated with TPC. In this article we propose RSSI based Optimum Transmission Power AODV (rOTP-AODV) which uses the concept of TPC in routing layer, so as to help nodes in minimizing net transmission power required to transmit the data from source to destination.

Rest of the article is organized in four sections. Section II summarizes the related work done on transmission power control techniques and other energy efficient routing protocols. Section III and IV details the system model and the proposed routing technique rOTP-AODV. The simulation of proposed routing technique is discussed in section V. Finally section VI draws the conclusion and future work.

\section{RELATED WORK}

The purpose of power-aware routing protocols is to maximize the network lifetime. Many algorithms have been proposed in order to save the energy of the network.

Gomez and Campbell in [1] analyzed the advantages of transmission power control in wireless multihop networks. They have derived an asymptotic expression for the average traffic carrying capacity of nodes in a multihop network, where nodes can individually control their transmission range. Authors showed that variable range transmission approach outperforms common range transmission approaches by about $50 \%$ in terms of power. They also demonstrated that traditional routing protocols which are based on common range transmission can only achieve about half the traffic carrying capacity compared to variable range approaches.

Ping Ding et al in DEMAC [2] have considered the effect of interference among the nodes in the network and on each of node state transitions. DEMAC adaptively calculates the optimal transmission power which avoids interference, minimizes the consumed energy and at the same time maximizes the throughput. It uses transmission power of RTS (Request to Send) to find the interference in the network, based on this interference, and the data payload, the receiver determines the optimal transmit power for the following transmission 
Xue Zhang et al proposed PCAR in [3], which is a power controlled routing protocol based on bellman ford routing algorithm and proportional integral power control [4]. PCAR adjusts the transmission power such that the derived topology is between Relative neighborhood graph (RNG) and Gabriel Graph (GG).

W. Chee et al in PMAR [5] proposes an on demand routing protocol which selects a route so as to maximize the minimum node battery and minimize the total transmission power required to reach the destination. As PMAR requires the route discovery phase to obtain information about all the possible routes, so the authors have also proposed a flood control mechanism. PMAR also requires the information about a nodes location to be readily available so it can only be used in location aware ad-hoc networks.

Gomez and Campbell in PARO [6] have suggested the use of redirectors to break long range transmission into set of smaller range transmission. One or more Redirectors are elected to forward packets on behalf of source- destination pair, thereby reducing the total transmission power consumed along the route. Yifei Wei et al in PC-AODV [7] propose a high data rate route selection scheme based on power control in physical layer. In PC-AODV a node first discovers the routes within the transmission range corresponding to the highest data rate, and the nodes keeps on increasing the transmission range to that corresponding to lower data rate until it finds a route or gets to the lowest data rate.

J. S. Yang et al [8] proposed a system, PAMP, based on the assumption that the source node knows the amount of power consumed in transmitting a data. Path discovery process is based on the remaining power of nodes recorded in the RREQ packet. After receiving the first RREQ, the power availability in the path is found. If the power available is not enough to complete data transmission, then the destination node waits for the later route requests to determine the path that has adequate power for the data transmission. After receiving all the RREQs, the destination node sends back the RREPs for all recorded paths to the source node. PAMP is better suited for the situation in which data transmission cannot be completed with only one path.

Suvarna P. Bhatsangave et al [9] proposed a routing algorithm, OAODV, in which the node does not forward RREQ unless there is enough energy left in node, and until the node density in its surrounding is more than a particular threshold. Optimized AODV analyzes these two parameters, when implementing routing discovery, and efficiently avoids the transmission of unnecessary information.

In essence, the Energy efficiency is a key factor in limiting the deploybility of Ad hoc networks. With the strict energy constraints in the Ad hoc networks, the energy consumed for data transmission, routes establishment and maintenance should be kept as low as possible. A lot of efforts have been put into this effect, many researchers have also worked on controlling the transmission power of the radio but that has been done on the network wide basis and more or less on the physical and medium access control (MAC) layer only. The proposed system brings the concept of transmission power control into the routing layer. However, the transmission power required for successful and error free communication depends heavily on the quality of the link between the communicating nodes. Therefore it becomes inevitable to take into account the link quality before tweaking the transmission power of the nodes. K. Srinivasan and P. Levis in [10] have worked on various link quality metrics, and they have showed that the RSSI has very small variance compared to LQI for any link over time suggesting that the RSSI in a single packet is a good estimate of the average RSSI over many packets in that link. Moreover, it is also clear that generally for RSSI values greater than $-85 \mathrm{dBm}$, packet reception ratio is at least $85 \%$ indicating a very good link. So the proposed algorithm uses RSSI as wireless link Quality metric.

\section{SYSTEM MODEL}

\subsection{Assumptions}

This paper considers a wireless ad-hoc network deployed for real life applications. The following assumptions have been made about the ad-hoc nodes and the network model.

\subsubsection{Nodes have adjustable transmission power} rOTP-AODV requires the nodes to have adjustable/ variable transmission power so that the nodes can tune to the various transmission power levels as dictated by algorithm.

\subsubsection{The links are symmetric}

This assumption dictates that the energy required to send data from one node to another node will be same as the energy required in sending same data in reverse direction.

\subsubsection{Substantial amount of data}

As the proposed system saves energy only while sending data after the route discovery, so it is evident that rOTP-AODV will be effective only if the number of control packets required is very less compared to the number of data packet to be transmitted.

The energy consumed in frequently changing the transmitting power of the node is very less.

All the control packets vis-à-vis RREQ, RREP, RERR, and RREP-ACK are always transmitted at default power level.

\subsection{Message Formats}

the proposed algorithm, rOTP-AODV is entirely based on AODV routing protocol so it uses same control messages, namely Route Request Packet (RREQ), Route Reply Packet (RREP), Route Error Packet (RERR) and Route Reply Acknowledgement (RREP-ACK) with some modifications. The section below briefly describes the message formats of the control packets.

\subsubsection{Route Request Packet format}

The RREQ packet in rOTP-AODV contains an extra field "Total Transmission Power Required (TtlTxPwrReq)" and it specifies the total transmission power required from source to the node currently handling the request.

\subsubsection{Route Reply Packet Format}

The RREP packet in IOTP-AODV contains two new fields. "Transmission Power Required (TxPwrReq)" which stores the transmission power required by the next hop for sending data to the node currently handling the RREP and vice versa. "Total Transmission Power Required (TtlTxPwrReq)", it is the total energy required for sending the packet from destination to the node currently handling the RREP.

\subsubsection{Route Error and Route Acknowledgment Packet Format}

RERR and RREP-ACK packets in rOTP-AODV have same format as do they have in AODV. 


\subsubsection{Route Table Entries}

The rOTP-AODV has slightly modified routing table with two new entries namely "OTP for next hop" which specifies the Transmission Power required to send a packet from current node to the next hop, and "total transmission power required" used to store the value of Total energy required to send the packet to the corresponding destination entry in the table.

\section{PROPOSED ALGORITHM}

The proposed algorithm, RSSI based optimum transmission power AODV (rOTP-AODV) intends to save energy by using variable transmission power. We have focused on the fact that for efficient and reliable communication/ transfer of data, there is no need for the sender node to transmit data at full transmitting power at all times. Efficient and reliable communication of data can be ensured even at lower transmission powers, provided the intended receiver receives the signal with power above certain threshold value (i.e. receiver sensitivity). Once the route has been established between the end points by AODV, the nodes along the route may have different distances between them, and hence the minimum power required to send data correctly will be different. Here proposed algorithm comes into action and asks the nodes to transmit data to next hop at certain calculated power level (i.e. Optimum Transmission Power) rather than transmitting at default/ maximum transmission power, thereby saving some amount of energy at every hop. In this way, said algorithm provides large scope for energy conservation. To implement the algorithm we need a metric to measure power of the received signals, for this purpose we have used RSSI.

\section{1 rOTP-AODV Route Discovery Process}

Like AODV, in rOTP-AODV as well the process of route discovery is divided into two phases. In first phase known as the Route Request phase, the source node that needs a valid route to the destination generates an RREQ with all its fields properly initialized and then broadcasts the RREQ. The RREQ generated by the originator is rebroadcasted by the intermediate nodes till it reaches the destination. Once the RREQ packet reaches the destination the second phase i.e. Route Reply Phase comes into action. In this phase, RREP packet generated by the destination is unicasted hop by hop back to the originator node and hence completing the Route Discovery Process. The section below briefly describes the Route Request Phase and Route Reply Phase.

\subsubsection{Route Request Phase}

a. The source node initiates the route discovery process by broadcasting the RREQ packets with Total Transmission Power Required initially set to zero.

b. Each node that receives a broadcasted RREQ packet, checks if it has previously received the RREQ with same flooding Id and originator node, if it has then this node silently ignores the newly received RREQ. If received RREQ has not yet been processed, then the node calculates following parameters.

The Optimum Transmission Power (OTP) value with which they have received the signal;

$$
\text { OTP }=\text { txPowerdefault }-(\text { RSSI }- \text { RxSensitivity })+\text { guard }
$$

The total transmission power required (TtlTxPwrReq) for the path traversed up to that node;

TtlTxPwrReq $=$ TtlTxPwrReq $+O T P$

c. Once the node calculates the OTP and Total Transmission Required, route table entries are made. The two calculated parameters, OTP and Total Transmission Power Required are also stored in the routing table as 'OTP for next hop' and 'Total Transmission Power Required' respectively along with other fields of the route table entries. Like AODV, rOTP-AODV also stores routes both for the source and the node from which this intermediate node has received the route request. In this way complete reverse path from current node to the originator node is formed at each hop.

d. Then the node currently handling the route request checks if it is the destination, if it's not the destination, then the current node rebroadcasts the RREQ. However Before further forwarding the RREQ packet, the 'Total Transmission Power Required' field of the RREQ packet is modified with the 'Total Transmission Power Required' calculated at the current node.

e. The process continues till the destination is reached. This forms the complete reverse path along with the values of OTP required from destination node to the originator node. This is the path that will be used to send route reply 'RREP' packet back to originator node

\subsubsection{Route Reply Process}

a. When the RREQ packet reaches the destination, the destination prepares a RREP packet and then following process takes place. destination checks for the next hop, towards the originator node, from the routing table towards the source and properly initializes the fields of RREP packet with both 'Transmission Power Required' and 'Total Transmission Power Required' initialized to 'OTP'. Finally the destination node unicasts RREP to the next hop towards the originator node.

b. When a node receives a RREP, it creates a route table entry for the forward route to destination along with the OTP for the hop from which it received the RREP and the 'Total Transmission Power Required'. If the current node is not the originator node then it fetches the route to the originator node from the route table and makes the corresponding changes in RREP for Transmission Power Required and Total Transmission Power Required as shown below:

$$
\begin{aligned}
& \text { TxPwrReq }=\text { OTP } \\
& \text { TtlTxPwrReq }=\text { TtlTxPwrReq }+ \text { OTP }
\end{aligned}
$$

The current node then forwards the modified RREP to the next hop as fetched form the route table.

c. The process continues till the RREP reaches the originator node thereby forming the complete forward path from source to the destination. Once the RREP packet reaches the originator node, and the originator node makes route table entries the route discovery process is complete. Now the source has the complete path to the destination for data transfer and each node has the required OTP value for next hop stored in its routing table.

\subsection{Data Transmission Process}

When the RREQ packet reaches the destination, the destination prepares a RREP packet and then following process takes place.

If an active route to the destination is not available in the routing table, then the node initiates route discovery process. 
If an active route to the destination is available in the routing table then

a. the node consult the routing table to fetch the next hop for the destination along with the required OTP

b. The node adjusts the transmission power of its transmitter equal to the OTP.

c. Node starts transmission of the Data to the next hop at the OTP.

When the data reaches any intermediate nodes, the intermediate node again checks its routing table for an active route to the destination and same process is repeated till the data reaches the destination.

\section{SIMULATION AND RESULTS}

The purposed algorithm rOTP-AODV has been simulated in Qualnet 5.1. We have compared its performance with AODV. Various simulations have been carried out to analyze the working of rOTP-AODV. Simulations have been performed with static nodes as well as mobile nodes. The section below describes the simulation environment used and various associated parameters.

\subsection{Scenario 1}

Here we have considered an Ad hoc Network having 20 static nodes (Figure 1) and rOTP-AODV as its routing protocol. Table below shows the parameters and their values.

TABLE I

Parameters of Scenario 1

\begin{tabular}{ll}
\hline \hline Parameter & Value \\
\hline Simulator & Qualnet 5.1 \\
No. of nodes & 20 \\
Simulation Time & $200 \mathrm{~s}$ \\
Environment Size & $1500 \times 1500 \mathrm{~m}^{2}$ \\
Transmission Power (Default) & $20 \mathrm{dBm}$ \\
Receiver Sensitivity & $-85 \mathrm{dBm}$ \\
Traffic Type & $\mathrm{CBR}(\mathrm{Constant}$ Bit Rate) \\
Packet Size & $512 \mathrm{~b}$ \\
Packet Rate & 10 packets/s \\
Mobility & Zero \\
Antenna Type & Omni directional \\
\hline \hline
\end{tabular}

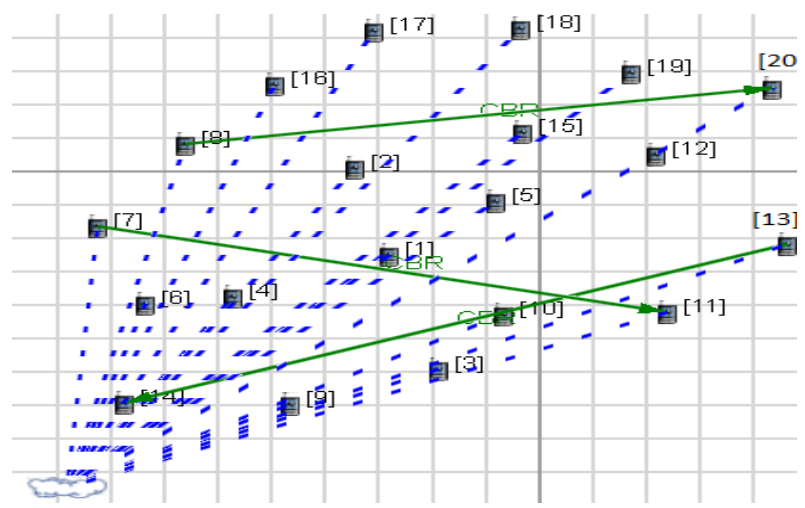

Figure 1: Screen Shot of Scenario 1

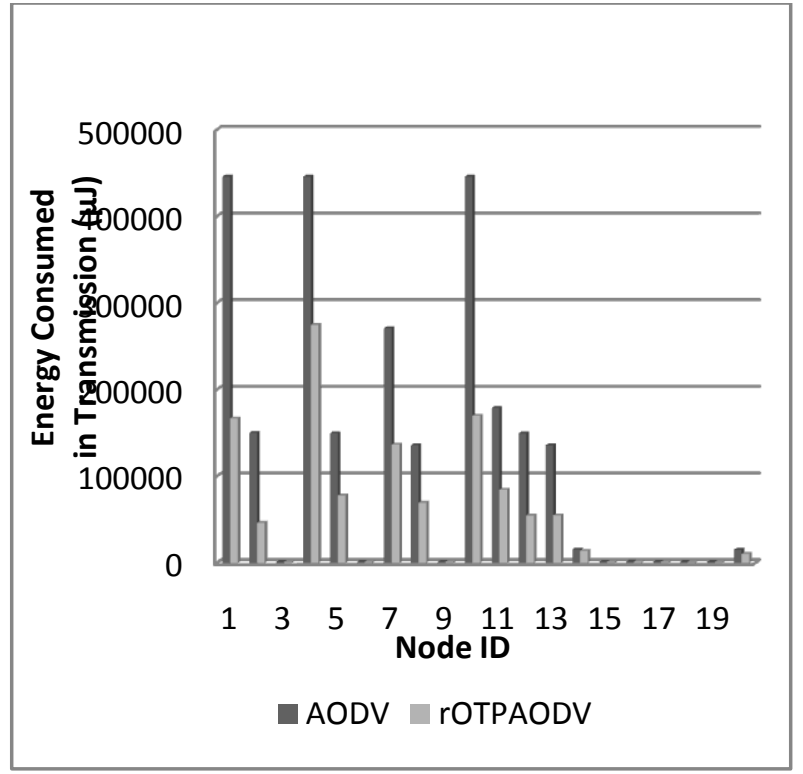

Figure 2: Comparison of energy consumed by nodes using AODV and rOTP-AODV

Figure 2 presents the comparison of energy consumed in transmission by nodes when using AODV and rOTP-AODV as routing protocol. From the chart it is evident that huge amount of energy is being saved when rOTP-AODV is used as compared to AODV. However it can also be noticed that the energy consumed by proposed algorithm rOTP-AODV is more than AODV if the number of data packets transmitted by the node is less than the number of control packets sent by same node. Further it may be understood that for same number of control packets to be transmitted, rOTP-AODV consumes more energy in transmission than AODV as the size of these control packets in rOTP-AODV is more than in AODV.

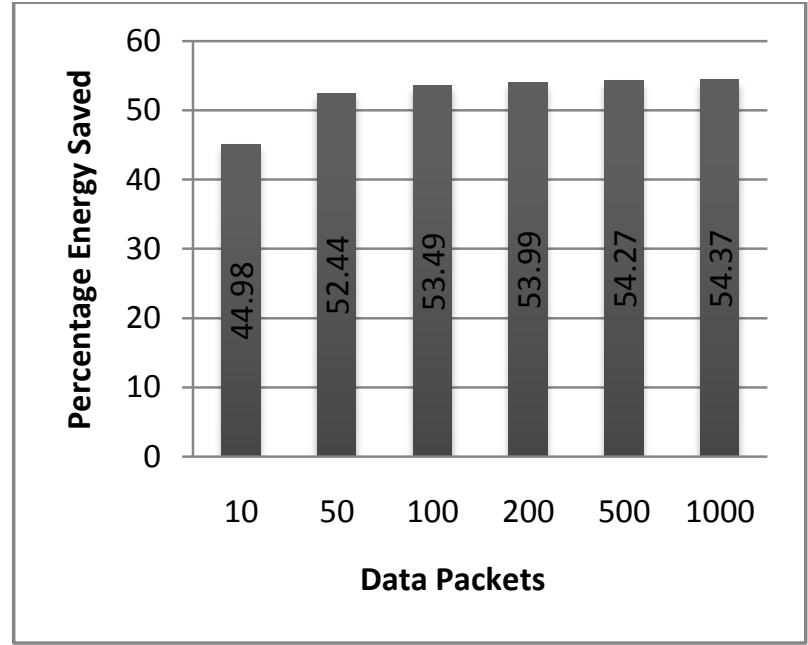

Figure 3: Percentage energy saved V/s no. of Data Packets (for each CBR)

The effect of number of data packets to be transmitted on the energy savings attained by rOTP-AODV has also been analyzed. Figure 3 presents the percentage of energy saved by rOTP-AODV when compared to the energy consumed by AODV routing protocol. The simulation results show that rOTP-AODV can save energy around 50\%. The simulation results also show that the percentage of energy saved 
increases with the increase in number of data packets sent over the CBR links.

\subsection{Scenario 2}

When we talk about wireless Ad hoc networks, mobility is one of the main features. Hence it becomes important to investigate the performance of rOTP-AODV in a network with mobile nodes. The nodes in scenario 2 obey random waypoint mobility. Other parameters associated with the simulation environment are shown in the table below.

\section{TABLE II}

Parameters Of Scenario 2

\begin{tabular}{ll}
\hline \hline Parameter & Value \\
\hline Simulator & Qualnet 5.1 \\
No. of nodes & 20 \\
Simulation Time & $200 \mathrm{~s}$ \\
Environment Size & $1500 \times 1500 \mathrm{~m}^{2}$ \\
Transmission Power (Default) & $20 \mathrm{dBm}$ \\
Receiver Sensitivity & $-85 \mathrm{dBm}$ \\
Traffic Type & $\mathrm{CBR}(\mathrm{Constant}$ Bit Rate) \\
Packet Size & $512 \mathrm{~b}$ \\
Packet Rate & $10 \mathrm{packets} / \mathrm{s}$ \\
Mobility model & Random Way Point \\
Speed & $1 \mathrm{~m} / \mathrm{s}$ \\
Pause Time & $2 \mathrm{~s}$ \\
Antenna Type & Omni directional \\
\hline \hline
\end{tabular}

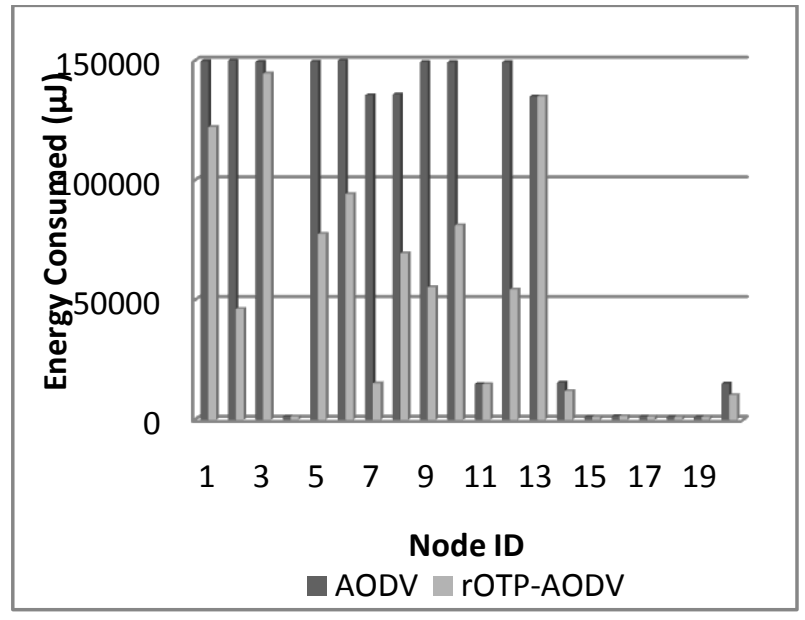

Figure 4: Comparison of energy consumed by nodes using AODV \& rOTP-AODV (Scenario 2)

Figure 4 presents the comparison of energy consumed in transmission by nodes when using AODV and rOTP-AODV as routing protocol for scenario 2 . In the current simulation environment $43 \%$ of energy is saved network wide. However when compared with energy saving in network with static nodes energy saving has reduced. This is because of the increased number of control packets flowing through the network owing to the link breakages caused by mobility.

To complete the comparison between AODV and rOTPAODV it is necessary that we analyze both the protocols on other quality of service indicators as well. In the section bellow we have compared AODV and rOTP-AODV on the basis of Throughput, End-To-End Delay, and Jitter.

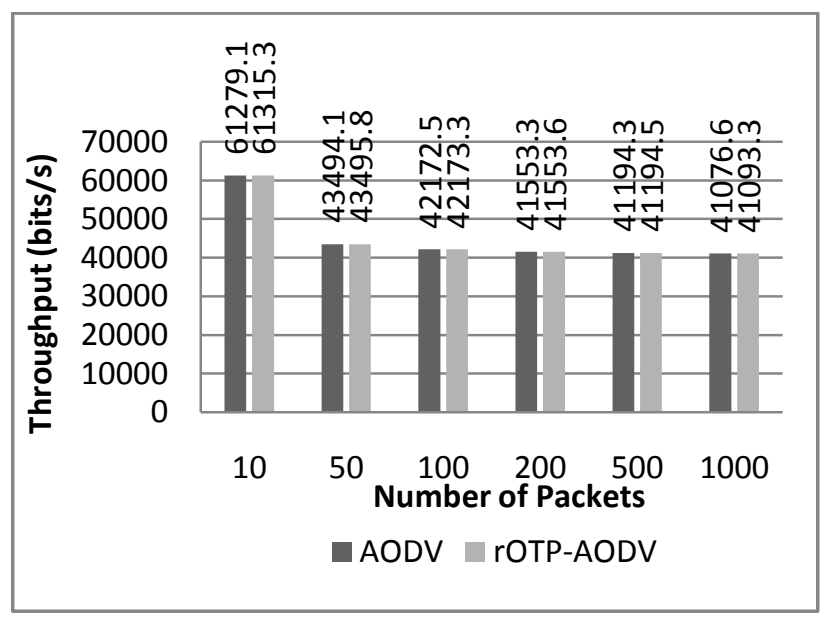

Figure 5: Comparison of throughput observed at nodes using AODV \& rOTP-AODV

Figure 5 depicts the comparison of throughput versus number of packets generated by each CBR when using AODV and rOTP-AODV as their routing protocol. It is quite evident that there is no adverse effect of rOTP-AODV on throughput, instead throughput has increased marginally.

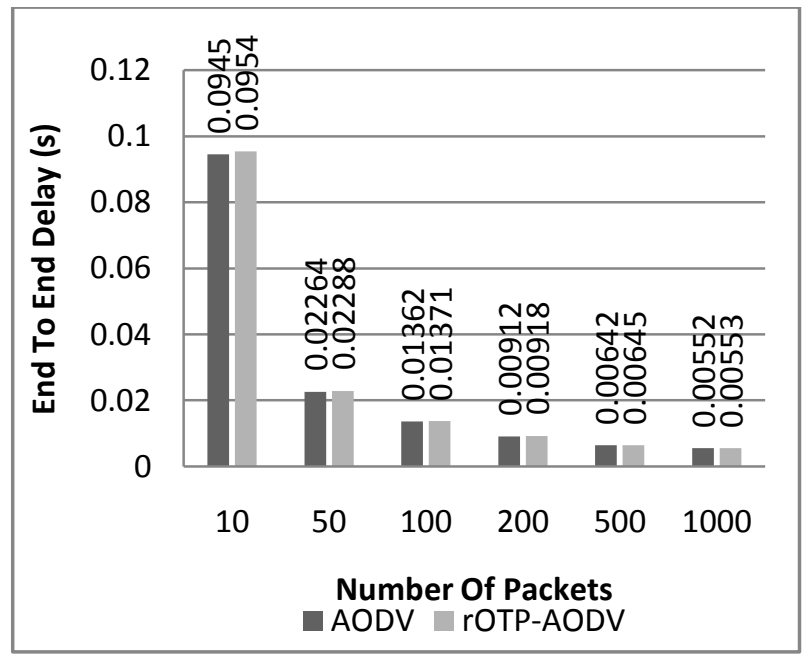

Figure 6 Comparison of End to End Delay observed at nodes using AODV \& rOTP-AODV

Figure 6 presents the comparison of AODV and rOTP-AODV on the basis of End to End Delay. Simulation results show that End To End delay has marginally increased $(0.6 \%)$ when rOTP-AODV was used. However such small increment in delay can be ignored in lieu of significant amount of energy consumed in transmission of data that can be saved by using rOTP-AODV. 


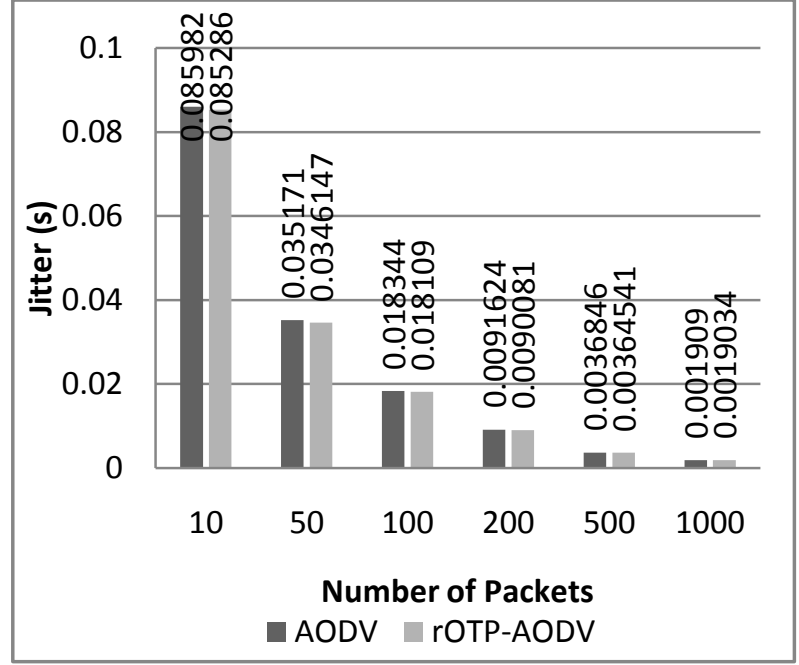

Figure 7: Comparison of Jitter observed at nodes using AODV \& rOTP-AODV

Figure 7 shows the comparison of AODV and rOTP-AODV on the basis of Jitter. As can be seen from the above figure, the jitter has slightly reduced $(1.1 \%)$ when we used rOTPAODV as routing protocol.

\section{CONCLUSION AND FUTURE WORK}

The proposed routing algorithm rOTP-AODV is an energy efficient algorithm which tries to conserve as much energy as possible while transmitting data between end points. The rOTP-AODV after route discovery process transmits data at optimum transmission power which each node has calculated and stored in routing table during route discovery process. By transmitting data at OTP considerable amount of energy can be saved.

Simulation results have shown that for wireless Ad hoc networks with static nodes as much as $50 \%$ of energy consumed in transmission can be saved thereby considerably extending the network lifetime. The amount of energy saved also increases as the number of data packets flowing through the network increases. However simulation results have shown and also justified our assumption that for rOTP-AODV to be effective the number of data packets should be very large compared to the control packets. Simulation results have also shown that for Mobile Ad-hoc networks the energy saved by rOTP-AODV is around $40 \%$. And the amount of energy saved in MANETs is bound to decrease with increase in mobility. The decrease in energy saved is due to the increased number of link breakages, which lead to more number of control packets flowing through the network.

Simulation results have also shown that rOTP-AODV does not adversely effects other major quality of service parameters such as Throughput, End to End Delay, and Jitter. In fact, except for End to End Delay which increased marginally $(0.6 \%)$ both other parameters Throughput and Jitter have improved with the use of rOTP-AODV over AODV. So we can say that proposed Algorithm i.e. rOTP-AODV performs better than AODV.
However currently the rOTP-AODV does not changes the process of route discovery, hence the concept of OTP is applied only to the routing path discovered by AODV. So the efficiency of rOTP-AODV can be further increased if we take total Transmission Power required into consideration while route formation.

\section{ACKNOWLEDGMENTS}

This work is the outcome of the sub component of UGC sponsored project titled "Secure and Quality of Service routing protocol in MANETS"

\section{REFERENCES}

[1] Javier Gomez and Andrew T. Campbell, "VariableRange Transmission Power Control in Wireless Ad Hoc Networks", in IEEE Transactions on Mobile Computing, Vol. 6, No. 1, pages 87-99, January 2007.

[2] Ping Ding, JoAnne Holliday and Aslihan Celik, "DEMAC: An Adaptive Power Control MAC Protocol for Ad-Hoc Networks", in IEEE 16th International Symposium on Personal, Indoor and Mobile Radio Communications, Vol. 2, pages 1389-1395, 2005.

[3] Xue Zhang, Ming Liu ; Haigang Gong, Sanglu Lu and Jie Wu, "PCAR: A Power Controlled Routing Protocol for Wireless Ad Hoc Networks", IEEE International Symposium on a World of Wireless Mobile and Multimedia Networks (WoWMoM), Pages 1-6, 2010.

[4] X. Zhang, Z. Li, S. Lu, D. Chen, and X. Li, "Proportionintegral power control for wireless ad hoc networks," in Proceedings of the IEEE Wireless Communications and Networking Conference (WCNC), Pages 2289- 2294, 2008.

[5] W. Chee Wah Tan, S.K. Bose and T.H. Cheng, "Power and mobility aware routing in wireless ad hoc networks", in IET Communications, Vol. 6, Issue 11, Pages 14251437, July 2012.

[6] Javier Gomez and Andrew T. Campbell, Mahmoud Naghshineh, Chatschik Bisdikian, "PARO: Supporting Dynamic Power Controlled Routing in Wireless Ad Hoc Networks", in Wireless Networks, Vol. 9, Issue 5, Pages 443-460, 2003.

[7] Yifei Wei, Mei Song and Junde Song, "An AODV Improved Routing Based on Power Control in $\mathrm{WiFi}$ Mesh Networks" in Canadian Conference on Electrical and Computer Engineering (CCECE), Pages 1349-1352, 2008.

[8] J. S. Yang, K. Kang, Y. J. Cho, S. Y. Chae, "PAMP: Power-Aware Multi-path Routing Protocol for a Wireless Ad-hoc Network", Proceedings of the IEEE WCNC, pages. 2247-2252, 2008

[9] Suvama p. Bhatsangave, V. R. chirchi "OAODV Routing Algorithm for Improving Energy Efficieny in MANET", International journal of Computer Applications, Volume 51, Issue 21, August 2012.

[10] K. Srinivasan and P. Levis, "RSSI is Under Appreciated", Proceedings of the Third Workshop on Embedded Networked Sensors, 2006. 